\title{
Triple Negative Breast Cancer-Review of Current and Emerging Therapeutic Strategies
}

Tarah Ballinger, Jill Kremer and Kathy Miller

Indiana University, School of Medicine, Division of Hematology/Oncology, Indianapolis, Indiana, US

DOI: https://doi.org/10.17925/OHR.2016.12.02.89

$\mathrm{T}$

riple negative breast cancer (TNBC) is associated with a poor prognosis compared to other types of breast cancer. The classification of 'triple negative' is not one homogenous tumor type, but rather is made up of multiple molecularly and biologically diverse tumor subtypes. At present, no approved targeted therapy exists and the standard remains cytotoxic chemotherapy. The identification of TNBC subtypes has provided a basis for identifying possible targeted therapeutic options. In addition, the recognition that some TNBCS share characteristics similar to tumors arising in patients with germline BRCA mutations has led to consideration of DNA damaging agents as a potential treatment option. Multiple investigational approaches are also underway, including immune checkpoint inhibition, poly (ADP-ribose) polymerase inhibition, and androgen receptor blockage. The limited options available for systemic treatment of TNBC will hopefully expand as more is learned about the complex biology and molecular targets of this group of breast cancers. This review will discuss the biology of TNBC, current treatment options, and promising experimental strategies.

\section{Keywords}

Triple negative, breast cancer, therapeutics

Disclosure: Tarah Ballinger, Jill Kremer and Kathy Miller have nothing to disclose in relation to this article. No funding was received in the publication of this article.

Authorship: All named authors meet the International Committee of Medical Journal Editors (ICMJE) criteria for authorship of this manuscript, take responsibility for the integrity of the work as a whole, and have given final approval to the version to be published.

open Access: This article is published under the Creative Commons Attribution Noncommercial License, which permits any noncommercial use, distribution, adaptation, and reproduction provided the original author(s) and source are given appropriate credit.

Received: September 9, 2016

Accepted: October 15, 2016

Citation: Oncology \& Hematology Review, 2016;12(2):89-94

Corresponding Author: Kathy Miller, Indiana

Cancer Pavilion, Suite 473, 535 Barnhill Dr,

Indianapolis, IN 46202-5289, US. E: kathmill@iu.edu
Triple negative breast cancer (TNBC) is defined by a lack of expression of estrogen receptor (ER) and progesterone receptor (PR), and a lack of overexpression or amplification of human epidermal growth factor 2 (HER2) on tumor cells. TNBC accounts for approximately 15-20\% of all breast cancers diagnosed in the United States. ${ }^{1}$ It is more common in African American women, younger women, and those with a germline BRCA1 mutation. Due to lack of ER, PR, and HER2 and the significant heterogeneity among TNBC, no approved targeted therapies exist and standard treatment remains cytotoxic chemotherapy. While response rates to chemotherapy in early stage disease are high, patients remain at high risk for relapse and prognosis remains inferior to other types of breast cancer.

\section{Subtyping and molecular characteristics}

In a seminal paper by Perou et al., gene expression profiling was used to categorize breast cancers into five molecular subtypes. The basal-like type is characterized by lack of ER, PR, and HER2 expression, expression of cytokeratins and EGFR, and a clinically more aggressive phenotype. ${ }^{2}$ This subtype overlaps with TNBC but the terms are not synonymous; in fact, about one fourth of TNBCs are not basal-like by gene expression, and some non-TNBCs are basal-like by molecular profiling (see Figure 1).3., As more is discovered about the biology of TNBC, it has become clear that this category of breast cancer is not one homogeneous tumor type, but rather is made up of a group of molecularly diverse tumor subtypes. These subtypes have varying gene expression profiles, clinical characteristics, and responses to treatment. In an effort to translate the heterogeneity of TNBC into rational clinical design, Lehmann and colleagues further characterized TNBC into six distinct subtypes based on molecular profiles, each with unique drivers and clinical phenotypes. These subtypes include basal-like 1 (BL1), basal-like 2 (BL2), immunomodulatory (IM), mesenchymal (M), mesenchymal stem-like (MSL), and luminal androgen receptor(LAR). ${ }^{5}$ Each exhibited different sensitivities to therapeutic agents, both in cell line models and in some retrospective clinical trials. For example, the BL1 subtype responds preferentially to platinum agents and responds significantly better to neoadjuvant chemotherapy than the BL2 subtype. The mesenchymal and luminal subtypes have greater sensitivity to phosphatidylinositol 3- kinase (PI3K) pathway inhibitors, while the LAR subtype is more sensitive to androgen receptor antagonists, with a relative insensitivity to standard chemotherapy. ${ }^{5-9}$

This heterogeneity and the lack of targetable oncogenic mutations have made the development of novel strategies for TNBC difficult. Common genomic alterations in TNBC result in dysregulated cell cycle progression and resistance to apoptosis, including the loss of TP53, RB1, BRCA1, and PTEN, as well as gain of function alterations in the cyclins/cyclin-dependent kinases (CCNE1, CCND1, CDK4, CDK6) and the PI3K pathway, including PIK3CA mutations. ${ }^{10-12}$ The most common of these alterations 
Figure 1: Venn diagram depicting the relationship between the triple negative immunophenotype and the basal-like molecular subtype

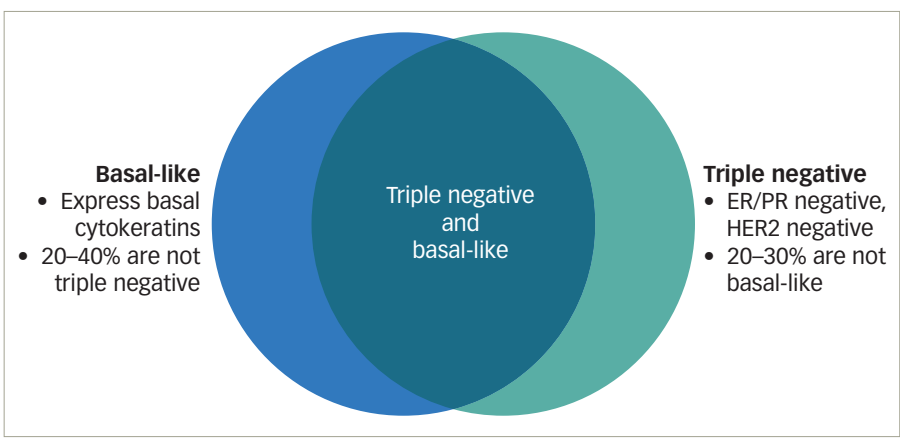

$E R=$ estrogen receptor; HER2 = human epidermal growth factor $2 ; P R=$ progesterone receptor.

is a mutation or loss of TP53, which is present in $68 \%$ of TNBCs and $80 \%$ of basal-like breast cancers in The Cancer Genome Atlas (TCGA), but is yet to have a targeted therapeutic agent. ${ }^{10}$

Further understanding of the biology of TNBC has come from the discovery that some sporadic tumors share similar characteristics with those tumors that have a germline mutation in the DNA repair gene BRCA1, which plays a role in DNA double-strand break repair by homologous recombination. This has led to the term 'BRCAness,' referring to genotypes of TNBC that are BRCA proficient but exhibit a clinical and biologic phenotype similar to those with BRCA deficiencies. There are likely alternative mechanisms leading to defects in homologous recombination in TNBCS without germline BRCA1/2 mutations, including promotor methylation, somatic BRCA mutations, and altered expression of other genes, such as TP53, PALB2, ATM, and HORMAD1. This phenotype has important implications, as TNBCs that harbor defects in homologous recombination have therapeutic sensitivities similar to those with BRCA mutations. ${ }^{13}$

\section{Current management of early stage disease}

Given the lack of approved targeted agents, the mainstay of treatment for TNBC is not unique and continues to be cytotoxic chemotherapy. All eligible patients with tumors over $0.5 \mathrm{~cm}$ in size should receive treatment with chemotherapy in either the neoadjuvant or adjuvant setting. There is no evidence favoring the use of neoadjuvant over adjuvant therapy in terms of outcome, but the neoadjuvant setting offers the advantage of having measurable disease to assess response to therapy, providing prognostic information. In addition, neoadjuvant therapy is the preferred approach for those patients who are not operable at diagnosis or who are not candidates for breast conserving therapy due to tumor size or location.

\section{Pathologic complete response}

TNBC has a higher response rate to neoadjuvant chemotherapy compared to breast cancers that are ER positive and a similar response rate to those that are HER2 positive. Those with TNBC who achieve a pathologic complete response ( $\mathrm{PCR}$ ) have improved outcomes compared to those who have residual disease. 14,15 In a pooled analysis by cortazar et al., there was an improvement in event free survival (EFS) (HR 0.24) and OS (HR 0.16) in patients with TNBC who achieved a pCR. However, pCR could not be validated as a surrogate endpoint for survival on a trial level analysis. ${ }^{15}$ In the neoadjuvant CALGB 40603 study, a pCR in the breast and axilla was associated with a $70 \%$ decrease in the risk of recurrence and an $80 \%$ decrease in the risk of death at three years. ${ }^{16}$ However, despite significantly more patients with TNBC achieving a PCR than those with luminal breast cancers, those with TNBC have a higher risk of recurrence. This paradox is likely due to the poorer prognosis of patients with residual disease. Residual disease in patients with TNBC confers a worse prognosis than residual disease in those with non-TNBC, with a significantly shorter recurrence free survival, decreased overall survival, and increased likelihood of developing visceral metastases. ${ }^{17,18}$

\section{Choice of regimen}

Similar to treatment of hormone positive or HER2 positive breast cancer, the preferred chemotherapy regimens in either the neoadjuvant or adjuvant setting contain an anthracycline and/or a taxane. The optimal regimen is not well defined and the choice of therapy ultimately depends on patient characteristics and preferences. There has been question of whether anthracyclines could be withheld in patients with HER2 negative breast cancer in the curative setting in order to avoid additional toxicity. A large pooled analysis by Gennari et al. in 2008 showed no added benefit in terms of disease-free or overall survival for the addition of an anthracycline to adjuvant chemotherapy. ${ }^{19}$ In addition, in a phase III trial by Jones et al. in 2006 comparing adjuvant doxorubicin plus cyclophosphamide (AC) to docetaxel plus cyclophosphamide (TC) in all types of breast cancer, disease-free survival (DFS) favored the TC arm and established this as an effective non-anthracycline choice. ${ }^{20}$ However, results recently presented at the 2016 American Society of Clinical Oncology (ASCO) annual meeting of the pooled 'ABC trials' (USOR 06-090, NSABP B-46I/USOR 07132, and NSABP $B-49)$ settled this question. The trials sought to evaluate whether TC was non-inferior to AC with a taxane. Results of the pooled analysis found that TC was not as effective as the regimens containing both an anthracycline and a taxane with a HR of 1.23 ( $p=0.04)$, or a $23 \%$ reduction in recurrence, favoring anthracyclines. The benefit was greatest in the HER2 negative, ER negative patients, with a HR of 1.42. ${ }^{21}$ While increased risk of heart failure and secondary leukemia should be discussed with patients, anthracyclines should not be withheld unless clinically necessary, and TC should not be considered an equivalent regimen in patients with TNBC.

\section{Role of additional agents}

Improving upon the anthracycline and taxane backbone has proven to be difficult. Despite initial promise for antiangiogenic therapy in breast cancer, phase III trials of the addition of the vascular endothelial growth factor (VEGF) inhibitor bevacizumab to adjuvant chemotherapy in TNBC failed to show improvement in disease-free or overall survival. ${ }^{22}$ In addition, the role of the addition of the antimetabolites capecitabine and gemcitabine remains unclear, with mixed results. Recently reported Iong-term outcomes from the FinXX trial of adjuvant capecitabine added to docetaxel, epirubicin, and cyclophosphamide showed no significant improvements in recurrence-free survival (RFS) or OS. While the subgroup analysis did how improvement for TNBC (RFS HR 0.54, OS HR 0.55), these results should be interpreted with caution given the small sample size. The US Oncology group trial 01062 also failed to show a DFS benefit when capecitabine was added to an anthracycline and taxane backbone. ${ }^{23}$ Furthermore, the addition of gemcitabine also did not improve outcomes in the NSABP B-38 study when added to adjuvant chemotherapy. ${ }^{24}$ In the Japanese CREATE-X trial, adjuvant capecitabine alone did improve DFS and OS in patients with HER2 negative disease who did not achieve a pCR after neoadjuvant anthracycline and taxane therapy, with the 
most impressive benefit being for patients with TNBC. ${ }^{25}$ The results are hypothesis-generating, and while capecitabine may be beneficial for a highly selective patient population, its role in early TNBC requires further study before routine clinical use.

\section{Role of platinum agents}

Similar to TNBCs arising in patients with germline BRCA mutations, many sporadic TNBCs also harbor defects in aspects of DNA repair, such as homologous recombination. ${ }^{26}$ Preclinical work has shown that BRCA1 deficiency predisposes tumors to be sensitive to platinum chemotherapy agents because the ability to repair DNA crosslinks created by platinum agents requires homologous recombination. Several neoadjuvant clinical trials have shown sensitivity to single agent platinum therapy, ${ }^{27.28}$ as well as increased pathologic complete response rates following neoadjuvant chemotherapy containing platinum agents. ${ }^{26,29}$ The largest of these is the CALGB 40603 study, in which 443 patients with stage II-III TNBC receiving a backbone of weekly paclitaxel for 12 weeks followed by dose-dense AC were randomized to also receive carboplatin, bevacizumab, or the combination of carboplatin and bevacizumab in a $2 \times 2$ design. The addition of carboplatin resulted in an improved PCR rate of $54 \%$, compared to $41 \%$ in the control group (OR 1.7). In the German GeparSixto study, 315 TNBC patients received paclitaxel with liposomal doxorubicin and bevacizumab with or without carboplatin. The addition of carboplatin resulted in an improvement in PCR from $37 \%$ to $53 \%$ (OR 1.94). The question of whether the increased $\mathrm{PCR}$ rates translate to an improvement in outcome remains unclear. In the Geparsixto study, the addition of carboplatin improved three-year DFS from $76.1 \%$ to $85.8 \%$ (HR 0.56); however, in the CALGB 40603 study, the improvement in EFS was less impressive and not significant $(71.6 \%$ to $76.5 \%$, HR 0.84$) .16,30$ This difference may be due to the fact that neither trial was powered for these survival endpoints. The GeparSixto study contained more node negative patients, used a nonstandard regimen, and used weekly carboplatin, rather than the everythree-week dosing used in CALGB 40603. In addition, it is important to note that toxicity is not trivial for the addition of carboplatin. Patients in the carboplatin arm of CALGB 40603 were more likely to have dose delays, miss treatments, or stop treatment early.

The ultimate benefit, mostappropriate regimen, and optimal dosing schedule for integration of platinum remains unknown, and we will await the results of ongoing clinical trials. The NRG-BR003 trial (NCT02488967) will evaluate the addition of carboplatin every three weeks to adjuvant AC followed by paclitaxel. ECOG-ACRIN 1131 (NCT02445391) will randomize patients with residual basal-like disease following neoadjuvant chemotherapy to either a platinum agent or capecitabine.

\section{Current management of metastatic disease}

The risk for distant recurrence of TNBC peaks at approximately three years, then rapidly declines. ${ }^{31}$ TNBC commonly recurs with visceral metastases in the lung and liver, with about 15\% of TNBC patients developing brain metastases. ${ }^{1}$ There is no current standard for the management of TNBC in the metastatic setting, and treatment continues to be a choice between cytotoxic chemotherapies depending on the specific patient. Given that the goal is palliation, individualized discussion with patients and consideration of the side effects or dosing schedules that will affect quality of life is essential. Few studies have focused on the TNBC subset alone in the metastatic setting, and the majority of data discussed here are not specific to triple negative disease.
Notably, there is no improvement in survival outcomes when chemotherapy agents are used in combination, compared to sequential use of single agents. ${ }^{32}$ However, for the highly selective patient who is deemed clinically to need a rapid tumor response to therapy in a short amount of time, combination therapy is reasonable. For patients who relapse later after primary therapy or present with de novo metastatic disease, it is reasonable to initiate therapy with a taxane. While docetaxel was shown in a randomized trial to have significant improvement in survival compared to everythree-week paclitaxel in the metastatic setting, docetaxel is associated with higher rates of toxicity, including myelosuppression. ${ }^{33}$ In addition, the preferred dosing of paclitaxel with weekly administration has never been compared to docetaxel in the metastatic setting. Docetaxel undergoes hepatic metabolism and therefore may not be a drug of choice for patients with significant liver tumor burden. Albumin bound nab-paclitaxel has the advantage of not requiring steroid pre-medication due to a decreased risk of allergic reaction with this formulation. However, in the randomized study CALGB 40502, there was no difference in outcome between weekly paclitaxel compared to weekly nab-paclitaxel used in the firstline setting, with more neuropathy and myelosuppression seen in the nab-paclitaxel arm. ${ }^{34}$ This study also included an arm with the non-taxane tubulin polymerizer ixabepilone, which proved inferior to paclitaxel. Most patients in this study also received bevacizumab; however, similar to outcomes in early breast cancer, the addition of bevacizumab to a taxane in the metastatic setting increases response rates and PFS, with no benefit in OS. ${ }^{35}$

Other choices include the anthracyclines, with the caveat that maximum lifetime anthracycline exposure is a limiting factor in patients with prior exposure in the neoadjuvant or adjuvant setting. Other active agents include the microtubule inhibitor eribulin, the antimetabolites gemcitabine and capecitabine, and the vinca alkaloid vinorelbine. Eribulin was compared to physicians' choice in patients with pretreated metastatic breast cancer, $20 \%$ of whom had TNBC, and resulted in significant improvement in OS (13.1 versus 10.6 months, HR 0.81), with neutropenia and peripheral neuropathy being significant side effects. ${ }^{36}$ This was the first study to show a benefit in pretreated patients using OS as the primary endpoint. A systematic review of capecitabine single agent therapy in patients with metastatic breast cancer and prior exposure to taxanes and anthracyclines showed a response rate of $18 \%$ and OS of 13.5 months. ${ }^{37}$ Capecitabine combinations have also been evaluated in the metastatic setting, with mixed results. Despite improvements in response rates and OS seen with some combinations, including docetaxel with capecitabine, the increase in toxicity has hindered adoption into routine clinical practice. ${ }^{38} \mathrm{~A}$ randomized study of eribulin versus capecitabine showed no statistical superiority of eribulin over capecitabine in PFS or OS ${ }^{39}$ however, a recently published exploratory, prespecified subgroup analysis did show a greater survival advantage with eribulin for patients with TNBC. ${ }^{40}$ Gemcitabine and vinorelbine also have activity as single agents in first line and pretreated metastatic breast cancer, with response rates of approximately $20-50 \%$, and both are commonly used due to favorable side effect profiles. ${ }^{4-44}$ Notably, gemcitabine has proven inferior to eribulin in the firstline metastatic setting. ${ }^{45}$

Similar to early-stage disease, the role of platinum agents in metastatic TNBC is promising but remains unclear. In the TNT trial of first line docetaxel versus carboplatin for metastatic TNBC, presented at the 2014 San Antonio Breast Cancer Symposium, there was no difference seen in response rates or survival. ${ }^{46}$ Among the BRCA1/2 positive patients, there was a $34.7 \%$ increase in response rates and significant improvement in PFS, but this 
Table 1: Results of clinical trials using immune checkpoint inhibitors in triple negative breast cancer

\begin{tabular}{|c|c|c|c|c|}
\hline Drug & Phase & Population & Number & Results in TNBC \\
\hline Pembrolizumab 50 & $\mathrm{Ib}$ & $\begin{array}{l}\text { Solid tumors, including heavily pre-treated } \\
\text { metastatic TNBC, PDL1 positive }\end{array}$ & $\begin{array}{l}27 \text { patients with TNBC evaluable } \\
\text { for efficacy }\end{array}$ & $\begin{array}{l}\text { ORR 18.5\% (1 CR, } 4 \text { PR), median duration of response not } \\
\text { reached (longest } 47 \text { weeks and ongoing) }\end{array}$ \\
\hline Atezolizumab 51 & la & $\begin{array}{l}\text { Solid tumors, including heavily pre-treated } \\
\text { metastatic TNBC }\end{array}$ & $\begin{array}{l}27 \text { patients with TNBC evaluable } \\
\text { for efficacy }\end{array}$ & $\begin{array}{l}\text { ORR 19\% ( } 2 \text { CR, } 2 \text { PR), median duration of response not } \\
\text { reached (longest } 84 \text { weeks and ongoing) }\end{array}$ \\
\hline Avelumab 52 & I & $\begin{array}{l}\text { Locally advanced or metastatic breast } \\
\text { cancer }\end{array}$ & 58 patients with TNBC & ORR for all patients $4.8 \%$ ( 5 of 8 responses were in TNBC) \\
\hline $\begin{array}{l}\text { Atezolizumab plus } \\
\text { nab-paclitaxe }{ }^{53}\end{array}$ & 1 & Metastatic TNBC & 32 patients evaluable for efficacy & $\begin{array}{l}\text { ORR } 46 \% \text { in } 1 \text { st line, } 22 \% \text { in } 2 \text { nd line, } 40 \% \text { in the } 3 \text { rd line } \\
\text { setting (0 CR, } 4 \text { PR) }\end{array}$ \\
\hline
\end{tabular}

$C R=$ complete response; $O R R=$ overall response rate; $P R=$ partial response; $T N B C=$ triple negative breast cancer.

subgroup contained only 43 patients. Overall, the majority of studies in the metastatic setting are underpowered and contain only small subsets of TNBC patients.

\section{Novel and targeted agents}

The transcriptional heterogeneity and lack of high-frequency mutations in TNBC add to the difficulty in creating targeted therapeutics. Interestingly, this can change under the influence of chemotherapy, which can increase the frequency of PTEN, PI3K, mTOR, and amplify JAK2, CDK6, CCND1, CCND2 and CCND3. ${ }^{12}$ For this subset of patients, there may be future prospects in targeted treatments.

\section{Immune checkpoint inhibitors}

The advent of immunotherapy and further understanding of the host immune system in highly proliferative cancers has opened opportunities in the study of TNBC. There is increasing evidence surrounding the role of tumor infiltrating lymphocytes (TILS) in TNBC. TILS have been associated with increased $\mathrm{pCR}$ rates and improved DFS and OS in TNBC. In the adjuvant BIG 02-98 trial, a 10\% increase in intratumoral or stromal lymphocytic infiltrate was associated with a $17 \%$ or $25 \%$ reduced risk of relapse, and a $27 \%$ or $17 \%$ reduced risk of death in TNBC, respectively. ${ }^{47}$ This and other outcome reports are suggestive of significant involvement of the immune system. ${ }^{48}$ Further research has found programmed cell death protein 1 ligand (PD-L1), which plays a role in inactivation of the host immune system, to be amplified in basal-like tumors and significantly correlated with the presence of $\mathrm{TILS}^{49}$ providing a rationale for targeting the PD1/ PD-L1 axis in TNBC. Two immune checkpoint inhibitors have been studied extensively in TNBC: the anti-PD-1 antibody pembrolizumab (MK-3475), and the anti-PD-L1 antibody atezolizumab (MPDL3280A). While there has been much excitement in the area of immunotherapy for TNBC, there is a relative paucity of data. The resulted clinic trials are detailed in Table 1, which have shown safety and clinical activity of single agents as well as in the combination of atezolizumab with nab-paclitaxel. ${ }^{50-53}$ While the PFS is short across these studies, patients who respond appear to have durable and ongoing responses. These compounds are being evaluated in larger trials in the neoadjuvant and metastatic setting. Additionally, studies of combinations of immunotherapy with chemotherapy are ongoing, including the phase III Impassion130 study of nab-paclitaxel plus atezolizumab in the first line metastatic setting (NCT02425891).

\section{Antibody drug conjugates}

Antibody drug conjugates (ADCS), which combine monoclonal antibodies with effector molecules and allow the cancer cell to internalize the antibody, are being studied for improved drug delivery and toxicity profile. One such ADC, glembatumumab vedotin (CDX-011 or CR011VCMMAE), is a combination of a gPNMB-specific monoclonal antibody and cytotoxin monomethyl auristatin E (MMAE). The glycoprotein gpNMB is overexpressed in TNBC and associated with increased risk of recurrence and metastases, while MMAE serves as a potent microtubule inhibitor. ${ }^{54,55}$ This ADC has been found to have enhanced activity against TNBC in early trials and is undergoing further investigation in larger trials such as EMERGE (NCT01156753) and METRIC (NCT01997333). Most recently, the ADC sacituzumab govitecan (IMMU-132), has been making headlines. This ADC is a combination of a moderately toxic drug SN-38, which is a topoisomerase I inhibitor that is the insoluble potent metabolite of irinotecan, and an antiTrop-2 monoclonal antibody targeting a glycoprotein found in TNBC. In a phase II study, the objective response rate was $31.5 \%$ with manageable toxicity, and the drug has been granted Breakthrough Therapy Designation and Fast Track Designation by the FDA in patients with TNBC following at least two previous treatments for metastatic disease..$^{56}$ Phase III research is pending (NCT02574455).

\section{Poly-ADP polymerase inhibitors}

Tumors arising in patients with BRCA1/2 mutations or those exhibiting a 'BRCAness' phenotype are characterized by deficiencies in homologous recombination DNA repair mechanisms, which has led to the evaluation of poly-ADP polymerase inhibitors (PARPi) in TNBC. PARP activation drives the synthesis of machinery involved in alternative mechanisms of DNA damage repair aside from homologous recombination. The rationale is that homologous recombination deficient cells will be reliant on PARP activation for DNA damage repair, and therefore particular sensitive to PARPi. Initial phase I and II data for BRCA- associated breast and ovarian cancers has shown promising results for PARPi as monotherapy, including veliparaib, olaparib, niraparib, talazoparib, and rucaparib, with response rates ranging from $0-50 \%$. There are also phase I and II studies of PARPi in combination with other agents in the metastatic setting showing efficacy in terms of response rates and clinical benefit. Table 2 highlights trial results for those multicenter trials that provide results for the BRCA mutated breast cancer population specifically. Trials in the non-mutated BRCA population are ongoing, hoping to identify a population with similar DNA repair defects. Ongoing phase III trials in the metastatic setting are evaluating PARPi monotherapy versus other single agents in patients with BRCA1/2 mutations (BRAVO NCT01905592, EMBRACA NCT01945775, and OlympiAD NCT02000622), and the study NCT02163694 is evaluating veliparib versus placebo in combination with carboplatin and paclitaxel. In the neoadjuvant setting, 
velparib in combination with carboplatin was recently found to have a PCR of $51 \%$ in patients with TNBC in the adaptive randomization trial I-SPY2, supporting further phase III study. ${ }^{57}$ The OlympiA trial of one year of olaparib in the adjuvant setting for patients with germline BRCA mutations is also ongoing (NCT02032823).

\section{Androgen receptor blockade}

The luminal androgen receptor (LAR) subtype of TNBC is characterized by chemoresistance, yet a lower risk of disease recurrence. ${ }^{67}$ It was hypothesized the AR inhibition would result in antitumor activity in this subtype of TNBC, a principle that was proved in the TBCRC001 phase II trial of bicalutamide in which 26 patients with AR positive, ER negative metastatic breast cancer had a $19 \%$ clinical benefit rate. ${ }^{8} \mathrm{~A}$ larger phase II trial of enzalutamide enrolling 118 patients with metastatic, AR positive TNBC showed a clinical benefit rate of $35 \%$ in evaluable patients. This study also showed that responses were better in those tumors with an androgen gene signature. ${ }^{68}$ These data support identifying patients with androgen receptor positivity and encourage additional clinical trials.

\section{Additional therapeutic targets}

An additional pathway under study in TNBC is the PI3K/AKT/mTOR signaling pathway, with basal-like breast cancers having a high frequency of activating PIK3CA mutations and IOSS Of PTEN. Clinical trials are underway in the phase II setting with AKT and PI3K inhibitors; however, due to the frequency of mutations in many other genes responsible for apoptosis, the antitumor effect as monotherapy in TNBC is likely limited. It is theorized that PI3K pathway inhibitors many increase DNA damage, as well as downregulate BRCA1/2, and therefore further sensitize tumor cells to PARPi. ${ }^{13}$ To evaluate this, a phase 1 trial of the pan-PI3K inhibitor BKM120 and olaparib is ongoing (NCT01623349). In addition, AR positive TNBCs are more likely to have PIK3CA mutations, and therefore antiandrogen plus PI3K inhibitor combinations are also being studied (NCT02457910).

Histone deacetylase (HDAC) inhibitors target heat-shock protein 90 (HSP90) that chaperones proteins like BRCA1/2. Early studies are evaluating these inhibitors in combination with chemotherapy, PARPi, and azacitidine (NCT02623751, NCT02393794, NCT01349959).

Another area of interest has been the growth factor FGFR. Pre-clinical work has shown decreased tumor growth with inhibition of FGFR in TNBCS with FGFR amplification. Several targeted agents are currently in development, and an ongoing trial is evaluating the tyrosine kinase inhibitor lucitanib in all breast cancers harboring an FGFR1 or 11q amplification (NCT02202746).
Table 2: Results of multicenter trial data for the use of poly-ADP polymerase inhibitors in BRCA mutated, metastatic breast cancer

\begin{tabular}{|c|c|c|c|}
\hline Drug & Phase & Population & Notable results \\
\hline Olaparib ${ }^{58}$ & $\|$ & $\begin{array}{l}\text { Recurrent, advanced } B C \text { with } \\
B R C A 1 / 2 \text { germline mutation } \\
(n=54)\end{array}$ & $\begin{array}{l}\text { ORR } 41 \% \text { at dose } \\
400 \mathrm{mg} \text { BID, ORR 22\% } \\
\text { at dose } 100 \mathrm{mg} \text { BID }\end{array}$ \\
\hline Olaparib 59 & $\|$ & $\begin{array}{l}\text { TNBC or ovarian cancer, } \\
\text { randomized by } B R C A \text { status } \\
\text { ( } \mathrm{n}=26 \text { with } \mathrm{TNBC}, \mathrm{n}=10 \text { with } \\
\text { BRCA mutation) }\end{array}$ & ORR $0 \%$ in $B C$ \\
\hline Olaparib ${ }^{60}$ & $\|$ & $\begin{array}{l}\text { Solid tumors with germline } \\
B R C A 1 / 2 \text { mutation, } B C \text { patients } \\
\text { with } \geq 3 \text { lines of prior therapy } \\
\text { ( } n=62 \text { with } B C \text { ) }\end{array}$ & $\begin{array}{l}\text { ORR } 12.9 \%, 47 \% \text { with } \\
\text { SD for } \geq 8 \text { weeks in } \\
\text { patients with BC }\end{array}$ \\
\hline $\begin{array}{l}\text { Olaparin plus } \\
\text { cisplatin }\end{array}$ & 1 & $\begin{array}{l}\text { Metastatic cancer }(\mathrm{n}=42 \text { with } \mathrm{BC} \text {, } \\
16 \text { with } B R C A \text { mutated } \mathrm{BC} \text { ) }\end{array}$ & $\begin{array}{l}\text { ORR } 71 \% \text { in BRCA } \\
\text { mutated } \mathrm{BC}\end{array}$ \\
\hline Veliparib ${ }^{62}$ & I & $\begin{array}{l}\text { Solid tumors with germline } \\
B R C A 1 / 2 \text { mutation or basal-like } \\
B C(\mathrm{n}=14 \text { with } B R C A \text { mutated } B C)\end{array}$ & $\begin{array}{l}\text { ORR } 29 \% \text { in BRCA } \\
\text { mutated BC, CBR } 57 \%\end{array}$ \\
\hline $\begin{array}{l}\text { Veliparib plus } \\
\text { carboplatin }\end{array}$ & 1 & $\begin{array}{l}\text { Metastatic } B C \text { with germline } \\
\text { BRCA1/2 mutation ( } n=26,70 \% \\
\text { of pts ER positive) }\end{array}$ & $\begin{array}{l}\text { ORR } 46 \% \text { (3 CR, } 9 \text { PR), } \\
\text { CBR 74\% }\end{array}$ \\
\hline $\begin{array}{l}\text { Veliparib plus } \\
\text { carboplain }{ }^{64}\end{array}$ & 1 & $\begin{array}{l}\text { Metastatic } \mathrm{BC} \text {, TNBC or mutation } \\
\text { in the Fanconi anemia pathway or } \\
\text { BRCA1/2 mutation ( } \mathrm{n}=44,16 \text { with } \\
\text { BRCA mutation) }\end{array}$ & $\begin{array}{l}25 \% \mathrm{PR}, 62.5 \% \mathrm{SD} \text { in } \\
\text { BRCA mutated } \mathrm{BC}\end{array}$ \\
\hline Talazoparib ${ }^{65}$ & I & $\begin{array}{l}\text { Metastatic solid tumor ( } \mathrm{n}=8 \text { with } \\
\mathrm{BC}, 6 \text { with } B R C A \text { mutation) }\end{array}$ & $\begin{array}{l}\text { ORR } 33 \% \text { in BRCA } \\
\text { mutated BC }\end{array}$ \\
\hline Niraparib 66 & I & $\begin{array}{l}\text { Metastatic solid tumor ( } \mathrm{n}=12 \text { with } \\
\mathrm{BC}, 4 \text { with } B R C A \text { mutation) }\end{array}$ & $\begin{array}{l}\text { ORR } 50 \% \text { in BRCA } \\
\text { mutated BC }\end{array}$ \\
\hline
\end{tabular}

$B C=$ breast cancer; $C B R=$ clinical benefit rate; $C R=$ complete response; $E R=$ estrogen receptor; $O R R=$ overall response rate; $P R=$ partial response; $S D=$ stable disease.

\section{Conclusions}

TNBC represents a heterogeneous group of breast cancers. Historically, a lack of molecular targets has left cytotoxic chemotherapy as the only treatment option for controlling systemic disease. However, as advancements are made in understanding the biology of TNBC, research is underway to understand the role of additional agents, including platinum agents, immune checkpoint inhibitors, PARP inhibitors, PI3K pathway inhibitors, and others. There is hope that the notoriously poor prognosis and limited number of options for TNBC will be transformed in the future by a personalized and targeted approach to treatment. $\mathrm{u}$
1. Anders CK, Carey LA, Biology, metastatic patterns, and treatment of patients with triple-negative breast cancer, Clin Breast Cancer 2009;9(Suppl 2):S73-81.

2. Perou $\mathrm{CM}$, Sorlie T, Eisen MB, et al., Molecular portraits of human breast tumours, Nature 2000:406:747-52.

3. Bertucci F, Finetti $\mathrm{P}$, Cervera N, et al., How basal are triple-negative breast cancers?, Int J Cancer, 2008;123:236-40.

4. Prat A, Adamo B, Cheang MC, et al., Molecular characterization of basal-like and non-basal-like triple-negative breast cancer, Oncologist, 2013;18:123-33.

5. Lehmann BD, Bauer JA, Chen X, et al., Identification of human triple-negative breast cancer subtypes and preclinical models fo selection of targeted therapies, I Clin Invest, 2011:121:2750-67.

6. Abramson VG, Lehmann BD, Ballinger TJ, et al., Subtyping of triple-negative breast cancer: implications for therapy, Cancer. 2015;121:8-16

7. Masuda $H$, Baggerly KA, Wang $Y$, et al., Differential response to neoadjuvant chemotherapy among 7 triple-negative breast cancer molecular subtypes, Clin Cancer Res, 2013;19:5533-40.

8. Gucalp A, Tolaney S, Isakoff SJ, et al., Phase II trial of bicalutamide in patients with androgen receptor-positive, estrogen receptor-negative metastatic Breast Cancer, Clin Cancer Res, receptor-negative mis:19:5505-12.

9. Lehmann BD, Jovanovic B, Chen $\mathrm{X}$, et al., Refinement of TripleNegative Breast Cancer Molecular Subtypes: Implications for Neoadjuvant Chemotherapy Selection, PLoS One, 2016;11:e0157368.

10. Cancer Genome Atlas N, Comprehensive molecular portraits of human breast tumours, Nature, 2012:490:61-70.

11. Shah SP, Roth A, Goya R, et al., The clonal and mutational evolution spectrum of primary triple-negative breast cancers, Nature, 2012:486:395-9.

12. Sharma P, Biology and Management of Patients With TripleNegative Breast Cancer, Oncologist, 2016
13. Bianchini G, Balko JM, Mayer IA, et al., Triple-negative breast cancer: challenges and opportunities of a heterogeneous disease Nat Rev Clin Oncol, 2016; [epub ahead of print].

14. von Minckwitz G, Untch M, Blohmer JU, et al., Definition and impact of pathologic complete response on prognosis after neoadjuvant chemotherapy in various intrinsic breast cancer subtypes, J Clin Oncol, 2012;30:1796-804

15. Cortazar P, Zhang L, Untch M, et al., Pathological complete response and long-term clinical benefit in breast cancer: the CTNeoBC pooled analysis, Lancet, 2014;384:164-72.

16. Sikov WM BD, Perou $C M$, et al., Event-free and overall survival following neoadjuvant weekly paclitaxel and dose-dense AC cancer: Outcomes from CALGB 40603 (Alliance). Proceedings of cancer: Outcomes from CALGB 40603 (Alliance). Proceedings of
the Thirty-Eighth Annual CTRC-AACR San Antonio Breast Cancer Symposium: 2015 Dec 8-12; San Antonio, TX. Philadelphia (PA): AACR, Cancer Res, 2016;76(4 Suppl):Abstract nr S2-05. 
17. Carey $L A$, Dees $E C$, Sawyer $L$, et al., The triple negative paradox: primary tumor chemosensitivity of breast cancer subtypes, Clin Cancer Res, 2007;13:2329-34.

18. Liedtke $C$, Mazouni $\mathrm{C}$, Hess KR, et al., Response to neoadjuvant therapy and long-term survival in patients with triple-negative breast cancer, I Clin Oncol, 26:1275-81.

19. Gennari A, Sormani MP, Pronzato P, et al., HER2 status and efficacy of adjuvant anthracyclines in early breast cancer: a pooled analysis of randomized trials, J Natl Cancer Inst, 2008;100:14-20

20. Jones SE, Savin MA, Holmes FA, et al.,Phase III trial comparing doxorubicin plus cyclophosphamide with docetaxel plus cyclophosphamide as adjuvant therapy for operable breast cancer, J Clin Oncol, 2006:24:5381-7.

21. Blum JL, Flynn PJ, Yothers G, et al., Interim joint analysis of the $\mathrm{ABC}$ (anthracyclines in early breast cancer) phase III trials (USOR 06-090, NSABP B-461/USOR 07132, NSABP B-49 [NRG Oncology]) comparing docetaxel + cyclophosphamide (TC) $\vee$ anthracycline/ taxane-based chemotherapy regimens (TaXAC) in women with high-risk, HER2-negative breast cancer. Program and abstracts of the 2016 American Society of Clinical Oncology Annual Meeting June 3-7, 2016; Chicago, Illinois. Abstract 1000.

22. Cameron D, Brown J, Dent R, et al., Adjuvant bevacizumabcontaining therapy in triple-negative breast cancer (BEATRICE): primary results of a randomised, phase 3 trial, Lancet Oncol, 2013;14:933-42.

23. O'Shaughnessy J, Koeppen $\mathrm{H}$, Xiao Y, et al., Patients with Slowly Proliferative Early Breast Cancer Have Low Five-Year Recurrence Rates in a Phase III Adjuvant Trial of Capecitabine, Clin Cancer Res, 2015;21:4305-11.

24. Swain SM, Tang G, Geyer CE, Jr., et al., Definitive results of a phase III adjuvant trial comparing three chemotherapy regimens in women with operable, node-positive breast cancer: the NSABP B-38 trial, J Clin Oncol, 2013;31:3197-204.

25. Toi M, Lee S-J, Lee ES, et al., A phase III trial of adjuvant capecitabine in breast cancer patients with HER2-negative pathologic residual invasive disease after neoadjuvant chemotherapy (CREATE-X, JBCRG-04). Program and abstracts of the San Antonio Breast Cancer Symposium; December 8-12, 2015: San Antonio, Texas. Abstract S1-07.

26. Le Du F, Eckhardt BL, Lim B, et al., Is the future of personalized therapy in triple-negative breast cancer based on molecular subtype?, Oncotarget, 2015;6:12890-908

27. Byrski T, Huzarski T, Dent R, et al., Response to neoadjuvant therapy with cisplatin in BRCA1-positive breast cancer patients, Breast Cancer Res Treat, 2009;115:359-63.

28. Silver DP, Richardson AL, Eklund AC, et al., Efficacy of neoadjuvant Cisplatin in triple-negative breast cancer, J Clin Oncol, 2010;28:1145-53.

29. von Minckwitz G, Schneeweiss A, Loibl S, et al., Neoadjuvant carboplatin in patients with triple-negative and HER2-positive early breast cancer (GeparSixto; GBG 66): a randomised phase 2 trial, Lancet Oncol, 2014;15:747-56.

30. von Minckwitz G, Loibl S, Schneeweiss A, et al., Early survival analysis of the randomized phase II trial investigating the addition of carboplatin to neoadjuvant therapy for triple-negative and HER2positive early breast cancer (GeparSixto). 2015 San Antonio Breast positive early breast cancer (GeparSixto). 2015 San Antonio Breast

31. Dent R, Trudeau M, Pritchard KI, et al., Triple-negative breast cancer: clinical features and patterns of recurrence, Clin Cancer Res, 2007;13:4429-34

32. Dear RF, McGeechan $\mathrm{K}$, Jenkins $\mathrm{MC}$, et al., Combination versus sequential single agent chemotherapy for metastatic breast cancer Cochrane Database Syst Rev 2013:CD008792.

33. Jones SE, Erban J, Overmoyer B, et al., Randomized phase II study of docetaxel compared with paclitaxel in metastatic breast cancer, J Clin Oncol, 2005;23:5542-51.
34. Rugo HS, Barry WT, Moreno-Aspitia A, et al., Randomized Phase III Trial of Paclitaxel Once Per Week Compared With Nanoparticle Albumin-Bound Nab-Paclitaxel Once Per Week or Ixabepilone With Bevacizumab As First-Line Chemotherapy for Locally Recurrent or Metastatic Breast Cancer: CALGB 40502/NCCTG NO63H (Alliance), J Clin Oncol, 2015:33:2361-9.

35. Miller K, Wang M, Gralow J, et al., Paclitaxel plus bevacizumab versus paclitaxel alone for metastatic breast cancer, N Eng/ I Med 2007;357:2666-76

36. Cortes J, O'Shaughnessy J, Loesch D, et al., Eribulin monotherapy versus treatment of physician's choice in patients with metastatic breast cancer (EMBRACE): a phase 3 open-label randomised study, Lancet, 2011:377:914-23.

37. Oostendorp $\sqcup$, Stalmeier PF, Donders AR, et al., Efficacy and safety of palliative chemotherapy for patients with advanced breast cancer pretreated with anthracyclines and taxanes: a systematic review, Lancet Oncol, 2011;12:1053-61.

38. O'Shaughnessy J, Capecitabine and docetaxel in advanced breast cancer: analyses of a phase III comparative trial, Oncology (Williston Park), 2002;16:17-22.

39. Kaufman PA, Awada A, Twelves $C$, et al., Phase III open-labe randomized study of eribulin mesylate versus capecitabine in patients with locally advanced or metastatic breast cancer previously treated with an anthracycline and a taxane, J Clin Oncol, 2015;33:594-601.

40. Twelves C, Awada A, Cortes J, et al., Subgroup Analyses from a Phase 3, Open-Label, Randomized Study of Eribulin Mesylate Versus Capecitabine in Pretreated Patients with Advanced or Metastatic Breast Cancer, Breast Cancer (Auckl), 2016:10:77-84.

41. Blackstein $\mathrm{M}$, Vogel $\mathrm{CL}$, Ambinder R, et al., Gemcitabine as firstline therapy in patients with metastatic breast cancer: a phase II trial, Oncology, 2002;62:2-8

42. Rha SY, Moon YH, Jeung HC, et al., Gemcitabine monotherapy as salvage chemotherapy in heavily pretreated metastatic breast cancer, Breast Cancer Res Treat, 2005;90:215-21.

43. Fumoleau P, Delgado FM, Delozier T, et al., Phase II trial of weekly intravenous vinorelbine in first-line advanced breast cancer chemotherapy, J Clin Oncol, 1993:11:1245-52.

44. Martin M, Ruiz A, Munoz M, et al., Gemcitabine plus vinorelbine versus vinorelbine monotherapy in patients with metastatic breast cancer previously treated with anthracyclines and taxanes: final results of the phase III Spanish Breast Cancer Research Group (GEICAM) trial, Lancet Oncol, 2007;8:219-25.

45. Feher $\mathrm{O}$, Vodvarka $\mathrm{P}$, Jassem J, et al., First-line gemcitabine versus epirubicin in postmenopausal women aged 60 or older with metastatic breast cancer: a multicenter, randomized, phase III study, Ann Oncol, 2005;16:899-908.

46. Tutt A, et al: The TNT trial. 2014 San Antonio Breast Cancer Symposium. Abstract S3-01. Presented December 11, 2014.

7. Loi S, Sirtaine N, Piette F, et al., Prognostic and predictive value of tumor-infiltrating lymphocytes in a phase III randomized adjuvant breast cancer trial in node-positive breast cancer comparing the addition of docetaxel to doxorubicin with doxorubicin-based chemotherapy: BIG 02-98, J Clin Oncol , 2013:31:860-7.

48. Anders CK, Abramson V, Tan T, et al., The Evolution of TripleNegative Breast Cancer: From Biology to Novel Therapeutics, Am Soc Clin Oncol Educ Book, 2016;35:34-42.

49. Ali HR, Glont SE, Blows FM, et al., PD-L1 protein expression in breast cancer is rare, enriched in basal-like tumours and associated with infiltrating lymphocytes, Ann Oncol, 2015;26:1488-93

50. Nanda R, Chow LQ, Dees EC, et al., Pembrolizumab in Patients With Advanced Triple-Negative Breast Cancer: Phase Ib KEYNOTE-012 Study, J Clin Oncol, 2016;34:2460-7.

51. Leisha A, Emens FSB, Cassier P, Inhibition of PD-L1 by MPDL3280A leads to clinical activity in patients with metastatic triple-negative breast cancer (TNBC) American Association of Cancer Research Philadelphia, PA, April 2015. Abstract 2859

52. Dirix LY, Takacs I, Nikolinakos P, et al., Avelumab (MSB0010718C) an anti-PD-L1 antibody, in patients with locally advanced or metastatic breast cancer: A phase Ib JAVELIN solid tumor trial. 2015 San Antonio Breast Cancer Symposium. Abstract S1-04. Presented December 9, 2015

53. Adams S, Diamond J, Hamilton E, et al., Phase Ib trial of atezolizumab in combination with nab-paclitaxel in patients with metastatic triple-negative breast cancer (mTNBC). Presented at: American Society of Clinical Oncology annual meeting. Chicago, Illinois. June 2016. Abstract 1009.

54. Bendell J, Saleh M, Rose AA, et al., Phase I/II study of the antibody-drug conjugate glembatumumab vedotin in patients with locally advanced or metastatic breast cancer, J Clin Oncol, 2014;32:3619-25.

55. Yardley DA, Weaver R, Melisko ME, et al., EMERGE: A Randomized Phase II Study of the Antibody-Drug Conjugate Glembatumumab Vedotin in Advanced Glycoprotein NMB-Expressing Breast Cancer, J Clin Oncol, 2015:33:1609-19.

56. An ADC for Triple-Negative Breast Cancer, Cancer Discov, 2016 Jan;6(1):OF8.

57. Rugo HS, Olopade OI, DeMichele A, et al., Adaptive Randomization of Veliparib-Carboplatin Treatment in Breast Cancer, N Engl J Med, 2016;375:23-34

58. Tutt A, Robson M, Garber JE, et al., Oral poly(ADP-ribose) polymerase inhibitor olaparib in patients with BRCA1 or BRCA2 mutations and advanced breast cancer: a proof-of-concept trial, Lancet, 2010:376:235-44.

59. Gelmon KA, Tischkowitz M, Mackay H, et al., Olaparib in patients with recurrent high-grade serous or poorly differentiated ovarian carcinoma or triple-negative breast cancer: a phase 2, multicentre, open-label, non-randomised study, Lancet Oncol, 2011;12:852-61.

60. Kaufman B, Shapira-Frommer R, Schmutzler RK, et al., Olaparib monotherapy in patients with advanced cancer and a germline BRCA1/2 mutation, J Clin Oncol, 2015:33:244-50.

61. Balmana J, Tung NM, Isakoff SJ, et al., Phase I trial of olaparib in combination with cisplatin for the treatment of patients with advanced breast, ovarian and other solid tumors, Ann Oncol 2014;25:1656-63.

62. Pahuja S, Beumer $\mathrm{JH}$, Appleman $\sqcup$, et al., Outcome of BRCA 1/2-mutated (BRCA+) and triple-negative, BRCA wild type (BRCAwt) breast cancer patients in a phase I study of single-agent veliparib M, J Clin Oncol, 2014:32:135.

63. Somlo G, Frankel PH, Luu TH, et al., Efficacy of the combination of ABT-888 (veliparib) and carboplatin in patients with BRCAassociated breast cancer, J Clin Oncol, 2013;31:1024.

64. Wesolowski R, Zhao M, Geyer SM, et al., Phase I trial of the PARP inhibitor veliparib $(V)$ in combination with carboplatin (C) in metastatic breast cancer (MBC), J Clin Oncol, 2014;32:1075.

65. De Bono JS, Mina LA, Gonzalez M, et al., First-in-human trial of novel oral PARP inhibitor BMN 673 in patients with solid tumors, J Clin Oncol, 2013:31:2580.

66. Sandhu SK, Schelman WR, Wilding G, et al., The poly(ADP-ribose) polymerase inhibitor niraparib (MK4827) in BRCA mutation carriers and patients with sporadic cancer: a phase 1 doseescalation trial, Lancet Oncol, 2013;14:882-92.

67. Wang C, Pan B, Zhu H, et al., Prognostic value of androgen receptor in triple negative breast cancer: A meta-analysis, Oncotarget, 2016

68. Traina TA, Miller K, Yardley DA, et al., Results from a phase 2 study of enzalutamide (ENZA), an androgen receptor (AR) inhibitor, in advanced AR+ triple-negative breast cancer (TNBC) J Clin Oncol, 2015;33:(suppl; abstr 1003). 